\title{
Estudo Prospectivo e Tecnológico da Geração de Syngas Utilizando Catalisadores
}

\author{
Prospective and Technological Study of Syngas Generation Using \\ Catalysis
}

\author{
Munique Gonçalves Guimarães ${ }^{1}$ \\ Grace Ferreira Ghesti ${ }^{1}$ \\ Camila Lisdalia Dantas Ferreira ${ }^{1}$ \\ ${ }^{1}$ Universidade de Brasília, Brasília, DF, Brasil
}

\begin{abstract}
Resumo
Com a crescente conscientização sobre o aquecimento global, o aumento de preço e a escassez dos combustíveis fósseis, torna-se necessário pesquisar e desenvolver fontes de energia alternativas. Uma possível fonte substituta é a biomassa, que tem grande quantidade de energia armazenada, mas perde boa parte desta para o ambiente nos processos de decomposição. O biogás é fonte de energia abundante, não poluidora, barata e que não compete com outras culturas e nem com a produção alimentícia. A partir desse contexto, o presente trabalho teve como objetivo apresentar um estudo prospectivo do estágio das pesquisas e da proteção patentária na área de biogás, syngas e catálise. Para tal, foram feitas pesquisas em bases de dados acadêmica e de patentes utilizando-se as seguintes combinações de palavras: "biogas", "syngas" e "syngas and cataly". Concluiu-se que as pesquisas sobre syngas e catálise apresentam baixa maturidade e que existe bastante espaço para desenvolvimento tecnológico nessa área, incentivado, muitas vezes, por políticas públicas específicas em diversos países.
\end{abstract}

Palavras-chave: Biogás. Catálise. Prospecção Tecnológica.

\begin{abstract}
With an awareness of global warming, rising prices and fuel shortages, alternative sources of energy are needed. Substitution is a biomass, which presents a large amount of energy lost during the decomposition process. Biogas is an abundant source of energy, non-polluting, cheap and non-competing for space with other crops or food production. Thus, the work aims to present a prospective study that portrays the research stage in the area. These were the word combinations for the study: "biogas", "syngas" and "syngas and cataly *". The work as research on the system and low schooling have a long nature and a technological incentive in the area of incentive in several countries.
\end{abstract}

Keywords: Biogas. Catalysis. Forecast.

Áreas tecnológicas: Biocombustíveis. Catálise. 


\section{Introdução}

Nos últimos anos, com a crescente conscientização sobre o aquecimento global - devido, principalmente, às emissões de gases de efeito estufa -, o aumento de preço e a escassez dos combustíveis fósseis, sentiu-se a necessidade de pesquisar e desenvolver fontes de energia alternativas, geradas a partir de recursos renováveis que apresentem alta eficiência, disponibilidade local para a geração de energia descentralizada e baixo nível de poluição (ZENG et al., 2018).

Nesse contexto, uma possível fonte substituta de combustíveis fósseis é a biomassa, que tem grande quantidade de energia armazenada, mas perde boa parte desta para o ambiente nos processos de decomposição: quando as bactérias retiram o alimento para sua sobrevivência, em contrapartida, lançam gás e calor na atmosfera. Esse gás é chamado biogás, uma fonte de energia abundante, não poluidora, barata e que não compete com outras culturas e nem com a produção alimentícia (SERRANO-LOTINA et al., 2011).

O biogás é produzido pela digestão anaeróbica, ou fermentação, de compostos orgânicos oriunda de diversas fontes, como: resíduos agrícolas, resíduos animais, lixo doméstico, resíduos industriais, esgoto (ZENG et al., 2018). Trata-se de um produto gasoso constituído por uma mistura de $\mathrm{CH}_{4}\left(55 \%\right.$ - 70\% v/v) e $\mathrm{CO}_{2},(27 \%$ - 44\% v/v) e, em menor quantidade, de outras impurezas, cuja concentração pode variar significativamente dependendo das características da biomassa, das condições operacionais, da disponibilidade de nutrientes, do biodigestor, do tempo de retenção, da taxa de degradação e outros (MORAL et al., 2018).

A composição do biogás afeta as possibilidades de seu aproveitamento, e uma elevada concentração de impurezas pode torná-lo energeticamente inviável (MORAL et al., 2018). Contudo, existem vários métodos disponíveis para limpeza de biogás, como absorção física, absorção, separação por membrana, separação criogênica e métodos químicos de conversão (KADAN; PANWAR, 2017).

Diante disso, o biogás tem se apresentado como uma fonte atrativa de carbono renovável e sua exploração pode ser vantajosa do ponto de vista tecnológico, uma vez que esse gás tem altos níveis de $\mathrm{CO}_{2}$ e $\mathrm{CH}_{4}$ (gases de efeito estufa), os quais, por meio de biodigestores, purificam-no convertendo-o em gás de síntese, ou syngas $\left(\mathrm{CO}\right.$ e $\left.\mathrm{H}_{2}\right)$, com a aplicação de um catalisador. $\mathrm{O}$ intuito desse tratamento é gerar produtos de alto valor agregado como na utilização da rota de reforma a seco que produz hidrocarbonetos substitutos do petróleo (MORAL et al., 2018).

Motivados pela possibilidade de geração de hidrocarbonetos com alto valor agregado, pela produção de energia descentralizada e por processos que sejam limpos em relação aos resíduos químicos gerados e que tenham baixo custo de produção, há um grande incentivo de diversos países para que estudos na área de catálise sejam conduzidos de forma que o processo se torne mais eficiente ambientalmente.

Os catalisadores são substâncias que alteram a velocidade de uma reação e devem atender a alguns pré-requisitos, como: seletividade em relação às reações desejadas, resistência à desativação, estabilidade térmica e mecânica. Além disso, devem apresentar características físicas importantes como: tamanho dos poros, área específica, localização da fase ativa, interação da fase ativa com o suporte, tamanho das partículas (ZENG et al., 2018).

Para a reforma de biogás, a principal dificuldade reacional está na desativação do catalisador, principalmente devido a fatores como: deposição de carbono na superfície, sinterização das 
partículas dos metais, fraca interação entre a fase metálica e o suporte, adsorção química forte de impurezas sobre os centros ativos do catalisador, transporte a vapor, atrito e esmagamento (DRIF et al., 2015). Por isso, o desenvolvimento de um bom catalisador inicia já na primeira etapa da reforma de biogás, com a escolha da fase ativa a ser empregada. Catalisadores metálicos são comumente utilizados em reações envolvendo hidrogênio e hidrocarbonetos, sendo o metal o principal componente do catalisador responsável pela ocorrência da reação química. Isso se deve ao fato de este tipo de substância adsorver facilmente em superfícies metálicas (HERMES, 2010).

Nesse sentido, pesquisas verificaram que catalisadores a base de metais nobres $(\mathrm{Ru}, \mathrm{Pt}, \mathrm{Pd}$ e Ir) são os mais indicados para a reforma de biogás, uma vez que apresentam boa atividade e alta resistência à deposição de coque. Entretanto, esses metais elevam os custos dos processos, devido ao alto custo e à pouca disponibilidade, inviabilizando seu uso. Por isso, os metais de transição dos grupos 8, 9 e 10 como Ni, Fe e Co são utilizados com frequência em substituição aos metais nobres, por serem bastante reativos, de baixo custo e apresentarem alta disponibilidade (LI et al., 2017).

Outra importante etapa do desenvolvimento de um bom catalizador é a escolha do suporte, a fim de alcançar elevada conversão de biogás. Os suportes são utilizados para aumentar a dispersão da fase ativa e o volume de poros, ampliar a área específica, melhorar a resistência mecânica e a estabilidade térmica e apresentar baixa sinterização, com o objetivo de inibir a deposição de carbono efetivamente e assim manter a atividade inicial e suas propriedades físico-químicas (YAO et al., 2017).

Diversos suportes são empregados na reforma do biogás, e os mais utilizados são: alumina ( $\mathrm{Al} 2 \mathrm{O} 3$ ) e sílica ( $\mathrm{SiO} 2)$, além de $\mathrm{MgO}, \mathrm{ZrO} 2$, óxidos de lantanídeos $\left(\mathrm{CeO}_{2}, \mathrm{LaO}_{2}, \mathrm{La}_{2} \mathrm{O}_{3}, \mathrm{Nd}_{2} \mathrm{O}_{3}\right)$, SBA-15, KIT-6, MCM-41, zeólitas e hidrotalcitas. (YAO et al., 2017)

Atualmente, as mudanças tecnológicas e as inovações estão cada vez mais presentes na vida das pessoas e nas grandes corporações. Por isso, a capacidade de realizar uma boa e adequada gestão do conhecimento tecnológico pode ser um diferencial para empresas que buscam a inovação e um diferencial no mercado. De acordo com Terra (2010 apud TEIXEIRA, 2013), o conhecimento tecnológico pode ser considerado um fator de vantagem igualmente importante quando comparado ao capital financeiro, físico, humano, aos recursos naturais e à localização de uma organização.

Assim, estudos prospectivos surgem como uma importante ferramenta de auxílio na gestão do conhecimento tecnológico. De acordo com Teixeira (2013), "[...] uma boa gestão tecnológica passa pelo entendimento da evolução do mercado de tecnologias, visando a antecipar possíveis novas tecnologias ou necessidades emergentes". A perspectiva apresentada por este trabalho pretende justamente entender a evolução das pesquisas e proteções patentárias nas áreas de biogás, syngas e catálise, para que seja possível identificar as tendências de mercado.

\section{Metodologia}

A metodologia utilizada baseou-se em duas perspectivas de prospecção em bases de dados: (i) tecnológica, realizada por meio de pesquisas de patentes; e (ii) científica, realizada por meio de pesquisa de artigos científicos. Para a prospecção tecnológica, utilizou-se a base de dados 
Orbit Intelligence ${ }^{\circledR}$; para a científica, a Web of Science ${ }^{\circledR}$. As buscas nessas bases de dados ocorreram no período de $1^{\circ}$ a 3 de julho de 2018.

$\mathrm{Na}$ base Orbit Intelligence ${ }^{\circledR}$, realizou-se uma busca avançada por patentes depositadas entre 1998 e 2017 que continham as combinações de palavras "biogas", "syngas", "(syngas and cataly*)" no título das patentes. A base Orbit Intelligence ${ }^{\circledR}$ também foi utilizada para a geração das figuras e dos gráficos que ilustram os resultados obtidos. Na base Web of Science ${ }^{\circledR}$, conduziu-se uma pesquisa básica utilizando-se os mesmos termos mencionados anteriormente no título de artigos científicos publicados entre 1998 e 2017. O principal operador booleano utilizado foi "and"; e o truncamento, “*”.

Por fim, realizou-se uma análise dos dados recuperados a partir da perspectiva mercadológica e da maturidade das pesquisas conduzidas, das tecnologias geradas e dos produtos identificados no mercado até o momento.

\section{Resultados e Discussão}

Para a condução da prospecção tecnológica e científica nas bases de dados referidas na metodologia, foram utilizadas as palavras-chave "biogas", "syngas" e a combinação "(syngas and cataly*)". Tais palavras foram selecionadas por satisfazerem critérios técnicos e científicos da área, sendo as que recuperaram documentos mais coerentes ao alcance dos objetivos deste estudo. A Tabela 1 mostra os resultados obtidos nas pesquisas de patentes e artigos científicos que serão discutidos no decorrer deste trabalho.

Tabela1 - Busca por combinação de palavras-chave nas bases Orbit Intelligence ${ }^{\circledR}$ e Web of Science ${ }^{\circledR}$

\begin{tabular}{ccc} 
Palavras-Chave & $\begin{array}{c}\text { Bancos Patentários } \\
\text { Orbit Intelligence }\end{array}$ & $\begin{array}{c}\text { Banco Científico } \\
\text { Web of Science }\end{array}$ \\
biogas & 6.549 & 5.458 \\
syngas & 1.133 & 3.466 \\
(syngas and cataly*) & 256 & 1.229 \\
\hline
\end{tabular}

Fonte: Elaborada pelas autoras deste artigo (2018)

Em linhas gerais, os resultados para a palavra-chave "biogas" mostraram que há mais patentes do que artigos científicos publicados, ao passo que, para as outras duas buscas, "syngas" e "(syngas and cataly*)", há mais artigos científicos publicados do que patentes. O resultado é importante para evidenciar a baixa maturidade das pesquisas sobre syngas e catálise e o espaço para desenvolvimento tecnológico que essa área possui.

\subsection{Biogás}

A presença acentuada de $\mathrm{CH}_{4}$ e $\mathrm{CO}_{2}$ no biogás torna sua utilização atrativa ambientalmente, por serem gases de efeito estufa (GEE) com potencial para reduzirem a poluição causada ao meio ambiente, além de serem um biocombustível alternativo, sustentável e amigavelmente correto (DOS SANTOS; DE SOUSA; PRATA, 2018). É comumente utilizado em comunidades 
isoladas e residências rurais como fonte para a obtenção de chama em fogões e para a proteção do solo, do ar e da vegetação lenhosa (SERRANO-LOTINA et al., 2011).

Por causa dos principais problemas relacionados ao uso direto do biogás em motores de combustão interna, principalmente devido a emissões tóxicas e à baixa eficiência gasosa, uma abordagem atrativa é convertê-lo usando a reforma catalítica, que tem o potencial para aproveitá-lo plenamente ao converter os gases de efeito estufa $\left(\mathrm{CH}_{4}\right.$ e $\left.\mathrm{CO}_{2}\right)$ em $\mathrm{H}_{2}$ e $\mathrm{CO}$, ou syngas (gás de síntese) (VITA et al., 2018).

A Figura 1 apresenta o resultado do levantamento do número de patentes e artigos encontrados com a palavra-chave "biogas". O objetivo da busca foi demonstrar quanto a tecnologia ainda está sendo pesquisada por empresas e universidades ao longo dos últimos 20 anos. Percebe-se até o ano de 2013 um número maior de patentes depositadas se comparado com o número de artigos científicos publicados.

Figura 1 - Quantidade de depósito de patentes e de publicação de artigos nos últimos 20 anos sobre "biogas", recuperados pela busca nas bases Orbit Intelligence ${ }^{\circledR}$ (azul) e Web of Science ${ }^{\circledR}$ (vermelho)

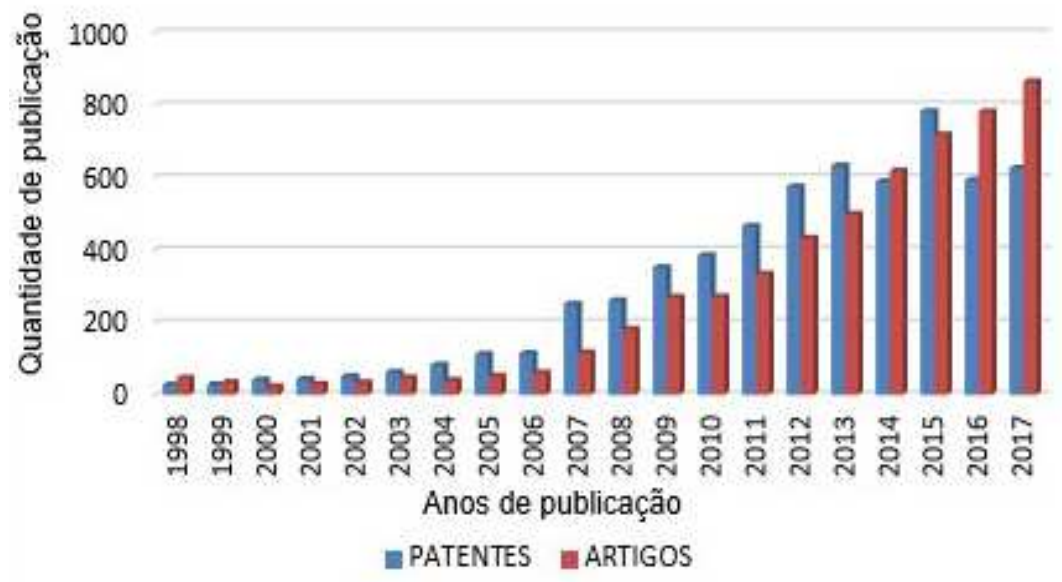

Fonte: Elaborada pelas autoras deste artigo (2018)

Tal resultado evidencia uma mudança de tendência de pesquisa aplicada para pesquisas mais básicas, induzidas, possivelmente, pela necessidade de se realizar mais estudos na área que busquem novas alternativas e abordagens de utilização do biogás.

A utilização do biogás como combustível contempla várias áreas tecnológicas, como agricultura, computação, alimentação. Do mesmo modo, existe uma ampla utilização de biogás em diversos domínios tecnológicos, conforme identificado nas consultas às plataformas Orbit Intelligence ${ }^{\circledR}$ e Web of Science ${ }^{\circledR}$. Todavia, percebeu-se nos resultados de ambas as consultas uma concentração nas seguintes áreas: basic materials chemistry, bio-technology, environmental technology, energy fuels, engineering, entre outras, conforme ilustra a Figura 2. 
Figura 2 - Patentes por domínio tecnológico que contenham a palavra "biogas"

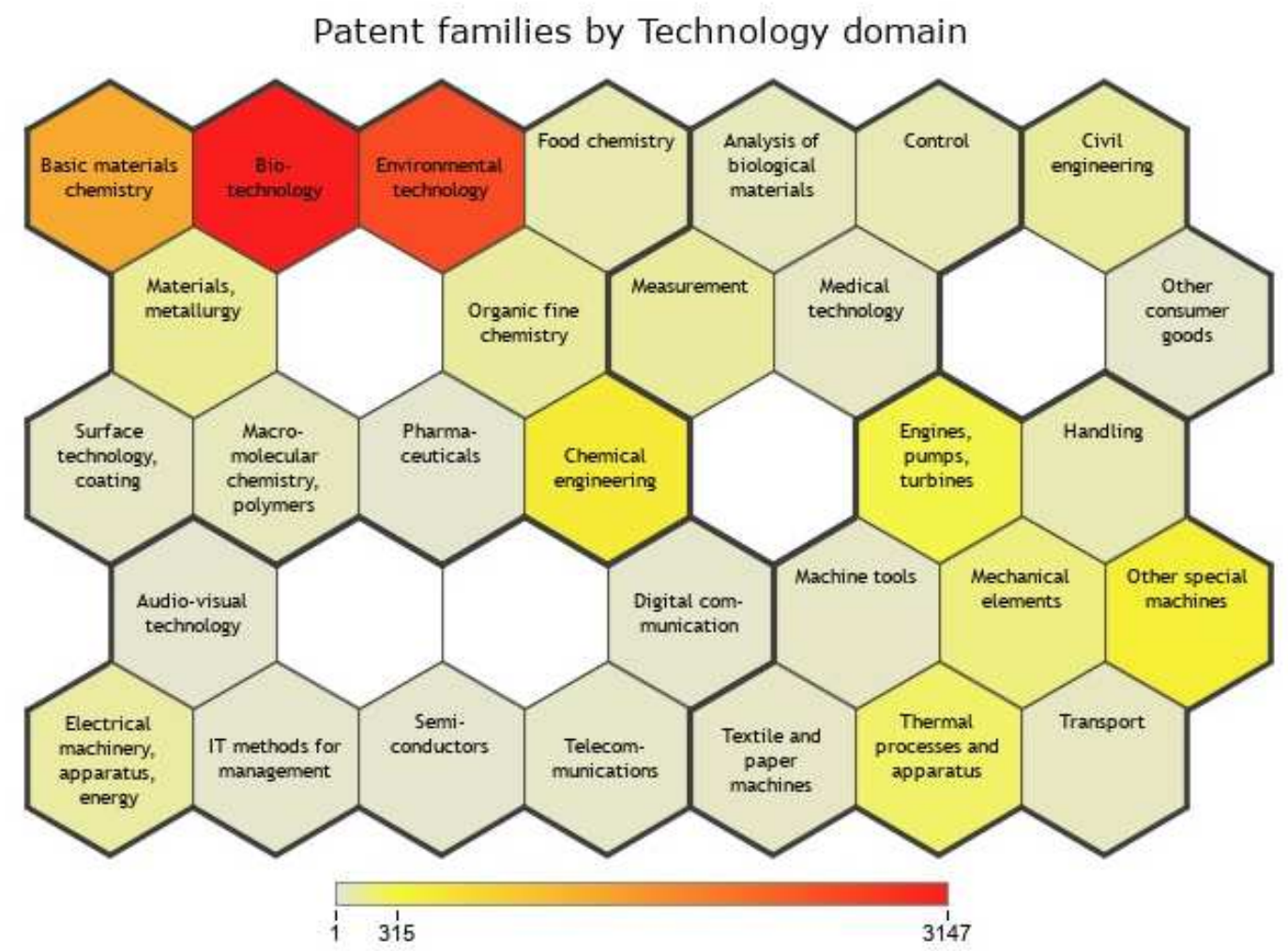

Fonte: Elaborada pelas autoras deste artigo, gerada no software Questel Orbit ${ }^{\circledR}$ (ORBIT INTELLIGENCE, 2018b)

Para o domínio tecnológico com maior concentração ("bio-technology"), foram identificadas 3.147 famílias de patentes, a maioria depositada na China, com 1.069 famílias, seguida pela Alemanha, com 227 famílias, e Espanha, com 178. O Brasil aparece com 23 famílias de patentes depositadas nesse domínio tecnológico. Importante destacar que os domínios mais presentes (basic materaisl chemistry, bio-technology e environmental technology) estão na área de geração de energia e processos mais ambientalmente recomendados, que envolve processos de design de reatores e sistemas de purificação de gás até modificação genética de micro-organismos para biodigestão.

\subsection{Syngas}

O syngas, ou gás de síntese, pode ser obtido de processos de conversão indireta do $\mathrm{CH}_{4}$. Existem três formas amplamente conhecidas para obtenção do syngas: (i) reforma do metano, que ocorre por meio da reforma a vapor (RV) (Equação 1); (ii) reforma a seco (RSM) (Equação 2); e (iii) oxidação parcial (OPPARCIAL) (Equação 3), que combina processos com a reforma autotérmica do metano. Além disso, o syngas também pode ser obtido do processo de gaseificação (PHAN et al., 2018).

(i) $\mathrm{RV}: \mathrm{CH}_{4(\mathrm{~g})}+\mathrm{H}_{2} \mathrm{O}(\mathrm{l}) \rightleftarrows \mathrm{CO}(\mathrm{g})+3 \mathrm{H}_{2(\mathrm{~g})}$ (Equação 1)

(ii) $\mathrm{RSM}: \mathrm{CH}_{4(\mathrm{~g})}+\mathrm{CO}_{2(\mathrm{~g})} \rightleftarrows 2 \mathrm{CO}_{(\mathrm{g})}+2 \mathrm{H}_{2(\mathrm{~g})}$ (Equação 2)

(iii) OPPARCIAL: $\mathrm{CH}_{4(\mathrm{~g})}+1 / 2 \mathrm{O}_{2(\mathrm{~g})} \rightleftarrows \mathrm{CO}(\mathrm{g})+2 \mathrm{H}_{2(\mathrm{~g})}$ (Equação 3) 
A literatura mostra que a reforma a vapor (RV) é eficiente para a conversão de biogás em syngas, com baixa taxa de desativação catalítica. A presença de vapor permite gaseificar a deposição de coque e limita assim a desativação catalítica. No entanto, a reforma a vapor é um processo que consome muita energia devido a sua alta temperatura operacional e alta taxa de produção de água. Já a oxidação parcial (OPPARCIAL) não permite a inclusão de $\mathrm{CO}_{2}$ no processo (PHAN et al., 2018).

Por essas razões, a reforma a seco do $\mathrm{CH}_{4}$ (RSM) tem sido intensamente investigada nos últimos anos como solução promissora para a reforma do biogás. O processo RSM desempenha um papel potencialmente importante na produção de syngas, especialmente devido aos baixos custos operacionais (isto é, $\sim 20 \%$ menor do que qualquer outro processo de reforma) e a possibilidade de utilizar gases com abundância de $\mathrm{CO}_{2}$ (PHAN et al., 2018). O syngas obtido a partir da reforma do biogás pode ser utilizado para sintetizar diversos produtos químicos, principalmente na produção de hidrocarbonetos líquidos, que são os principais subprodutos do petróleo.

Ao realizar a busca patentária utilizando-se a palavra-chave "syngas", obteve-se o resultado de 2.675 patentes publicadas nos últimos 20 anos. A Figura 3 mostra a evolução dos depósitos na área, por ano, sendo o crescimento dos depósitos bastante expressivo a partir de 2012.

Figura 3 - Patentes por ano de publicação utilizando-se a palavra-chave"syngas"

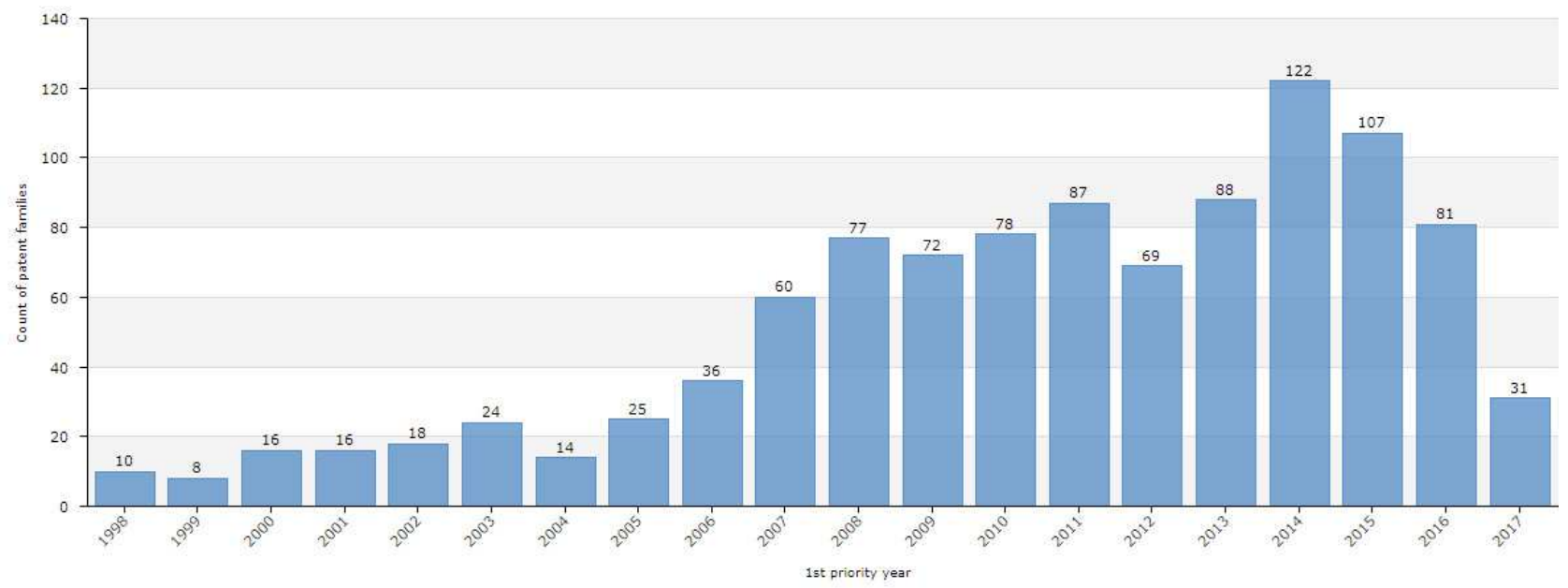

Fonte: Elaborada pelas autoras deste artigo, gerada no software Questel Orbit@ (ORBIT INTELLIGENCE, 2018b)

Além da concentração de depósitos registrados nos últimos 20 anos, também foi identificado um número significativo de depósitos internacionais via Tratado de Cooperação em Matéria de Patentes (PCT) oriundos, em sua maioria, de grandes corporações como General Electric e China Petroeum \& Chemical. A General Electric é um conglomerado multinacional americano que desenvolve produtos em diversas áreas, entre elas petróleo \& gás e energia renovável. Essa empresa tem uma intensa atividade de Pesquisa \& Desenvolvimento (P\&D) com gaseificadores em geral e até mesmo possui plantas de produção de energia limpa por meio de turbinas de gás chamadas Integrated Gasification Combined-Cycle (IGCC). 
Figura 4 - Principais empresas depositantes no setor de energia renovável

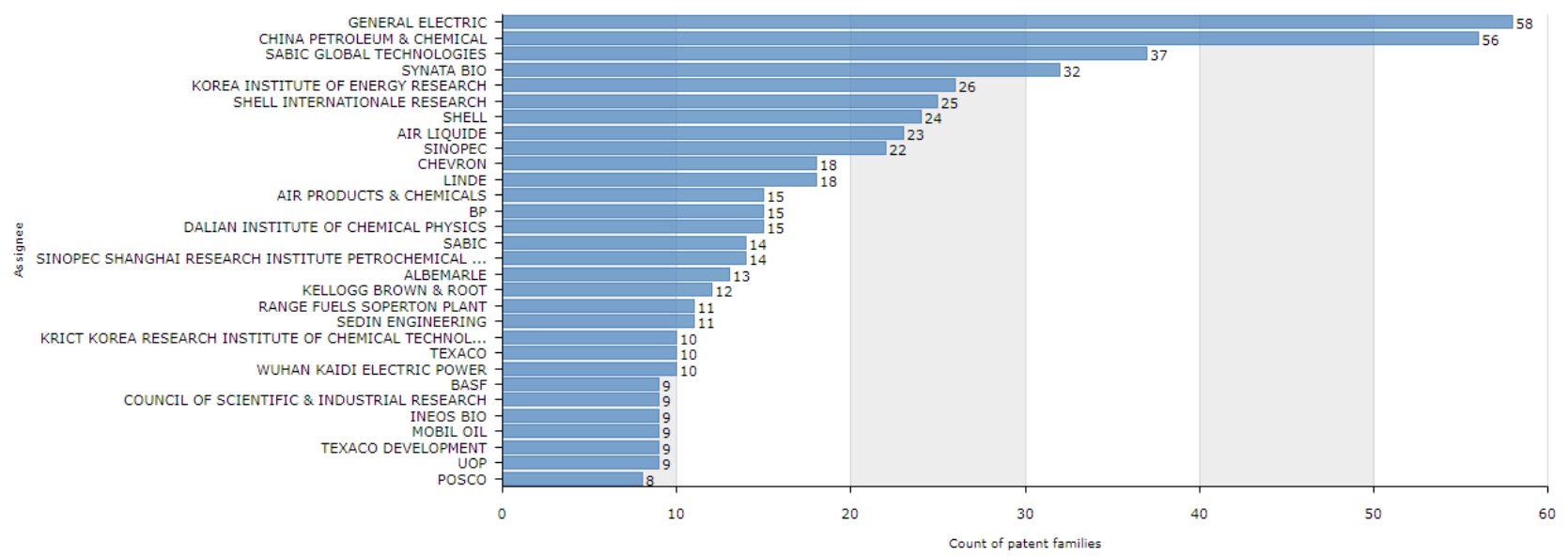

Fonte: Elaborada pelas autoras deste artigo, gerada no software Questel Orbit@ (ORBIT INTELLIGENCE, 2018b)

A China Petroleum \& Chemical é uma empresa chinesa fornecedora de produtos químicos e derivados do petróleo, com experiência em exploração onshore e offshore de óleo cru e gás natural, processamento, refino, distribuição, transporte e comercialização.

Tanto a China Petroleum \& Químical quanto a General Electric são líderes no setor e certamente têm induzido as pesquisas na área de produção de syngas.

Ademais, conforme ilustra a Figura 5, existe uma grande quantidade de aplicações para a tecnologia do syngas. Dentre elas se destacam o syngas e o uso de catalisadores para produção de subprodutos de alto valor agregado como metanol e hidrocarbonetos leves.

Figura 5 - Aplicações tecnológicas do syngas

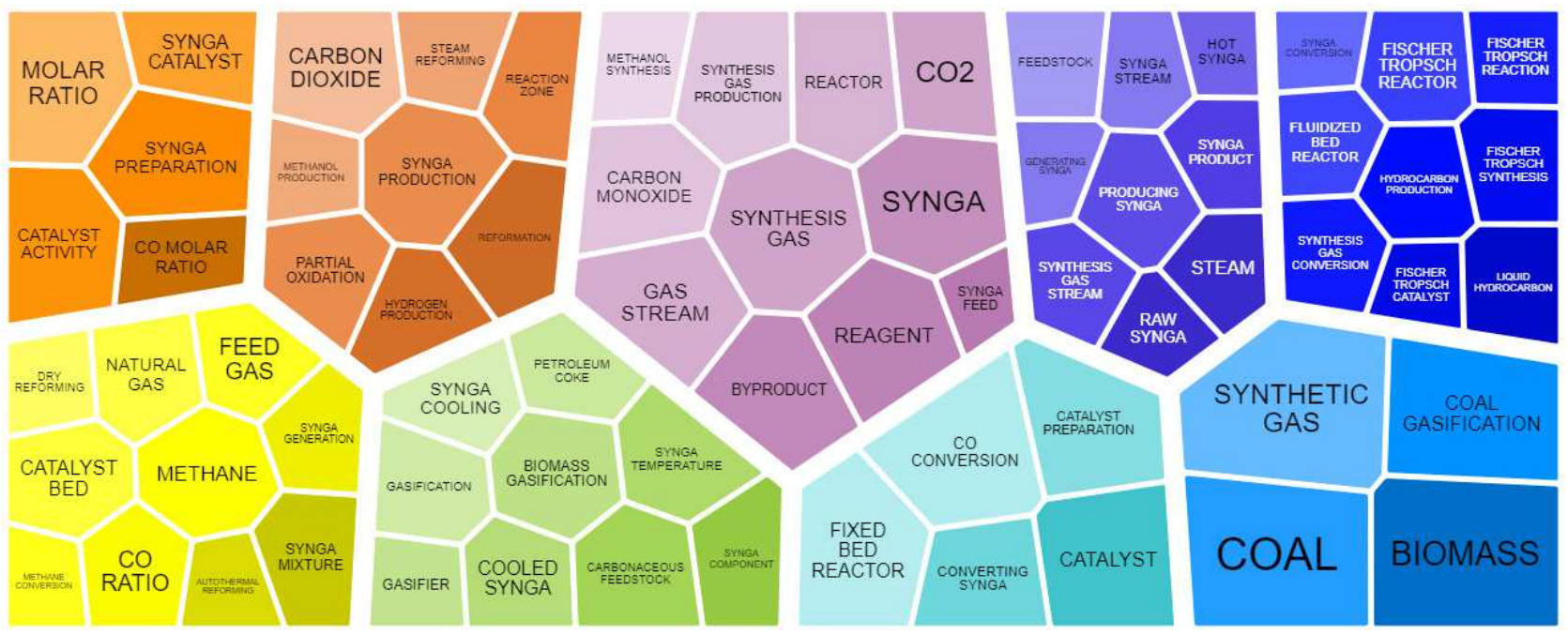

Fonte: Elaborada pelas autoras deste artigo, gerada no software Questel Orbit®) (ORBIT INTELLIGENCE, 2018b) 
No entanto, tanto o $\mathrm{CO}_{2}$ quanto o $\mathrm{CH}_{4}$ são moléculas estáveis, portanto é necessário a presença de altas temperaturas reacionais $\left(>700^{\circ} \mathrm{C}\right.$ ) para quebrar a barreira termodinâmica dessa reação, que é endotérmica. Uma possível alternativa à presença de elevadas temperaturas é a utilização de catalisadores que influenciam na formação dos produtos que podem variar entre a produção de metano e a de hidrocarbonetos de maior peso molecular como olefinas e parafinas (SCHULTZ; SOARES, 2014)

\subsection{Syngas e Catálise}

Com o objetivo de refinar ainda mais a pesquisa, foi conduzida, inicialmente, uma busca patentária com a palavra-chave "syngas", da qual se obteve um total de 1.134 documentos. Verificou-se que as atividades de P\&D relacionadas ao tema estão fortemente concentradas na China, nos Estados Unidas e na União Europeia. O Brasil apareceu em $13^{\circ}$ posição com 61 famílias patentes depositadas. A Figura 6 retrata essa realidade.

Figura 6 - Países que mais publicam sobre o tema syngas

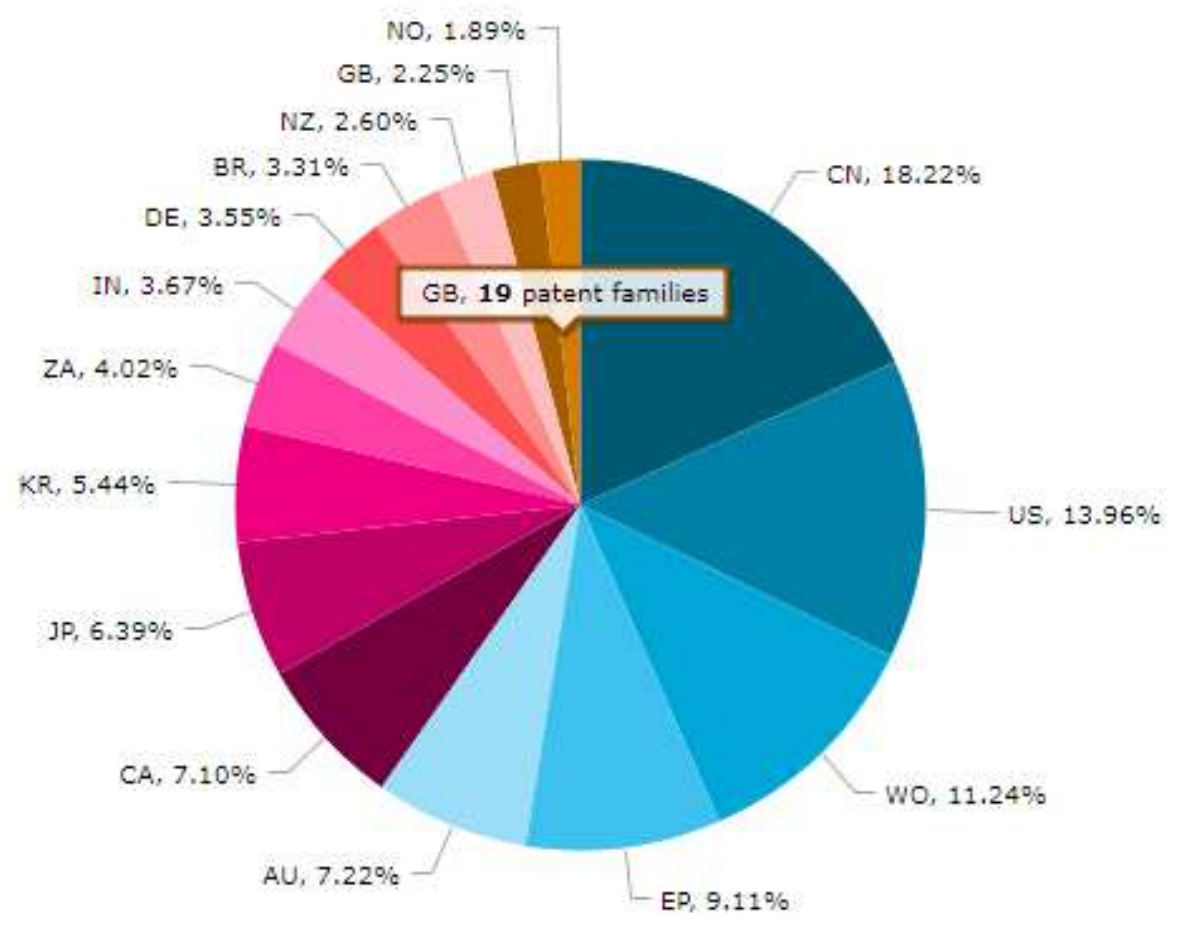

Fonte: Elaborada pelas autoras deste artigo, gerada no software Questel Orbit ${ }^{\circ}$ (ORBIT INTELLIGENCE, 2018b)

Dentre as empresas que mais desenvolvem patentes na área, destacam-se grandes multinacionais como a China Petroleum \& Chemical (ou China Sinopec), conforme mostrado na Figura 7. Na prospecção também foram identificadas outras grandes empresas como General Electric e Shell, com depósitos em números bem inferiores, mas que não deixam de indicar uma tendência de investimentos em P\&D no setor. 
Figura 7 - Empresas que mais depositaram patentes na área de syngas

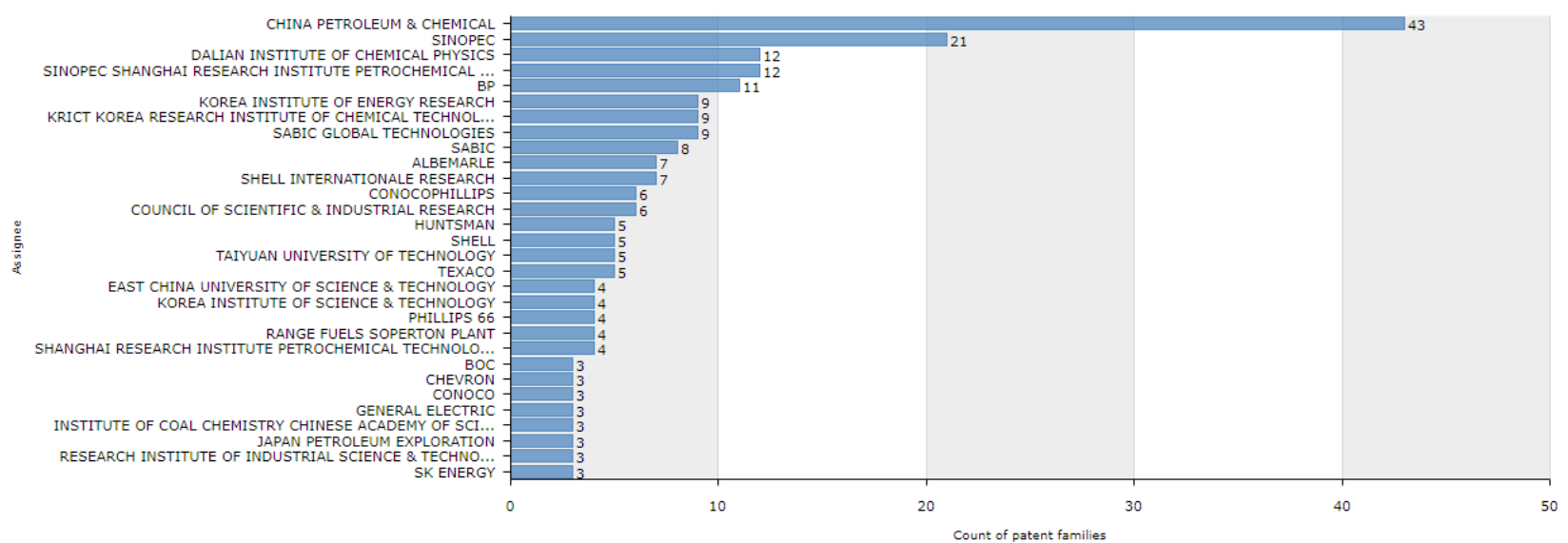

Fonte: Elaborada pelas autoras deste artigo, gerada no software Questel Orbit@ (ORBIT INTELLIGENCE, 2018b)

Por fim, foi inserido o termo "cataly*", pois o uso de catalisadores aplicados ao syngas pode gerar diversos subprodutos de alto valor agregado, sendo importante refinar os resultados para saber a catálise envolvida no processo. Desse modo, a combinação de palavras-chave "(syngas and cataly*)" resultou 256 pedidos de patentes. O baixo número de patentes reforça a incipiência de pesquisas na área, que passaram a gerar resultados mais expressivos apenas a partir de 2014, quando o número de depósitos aumenta significativamente (Figura 8).

Figura 8 - Quantidade de depósito de patentes nos últimos 20 anos

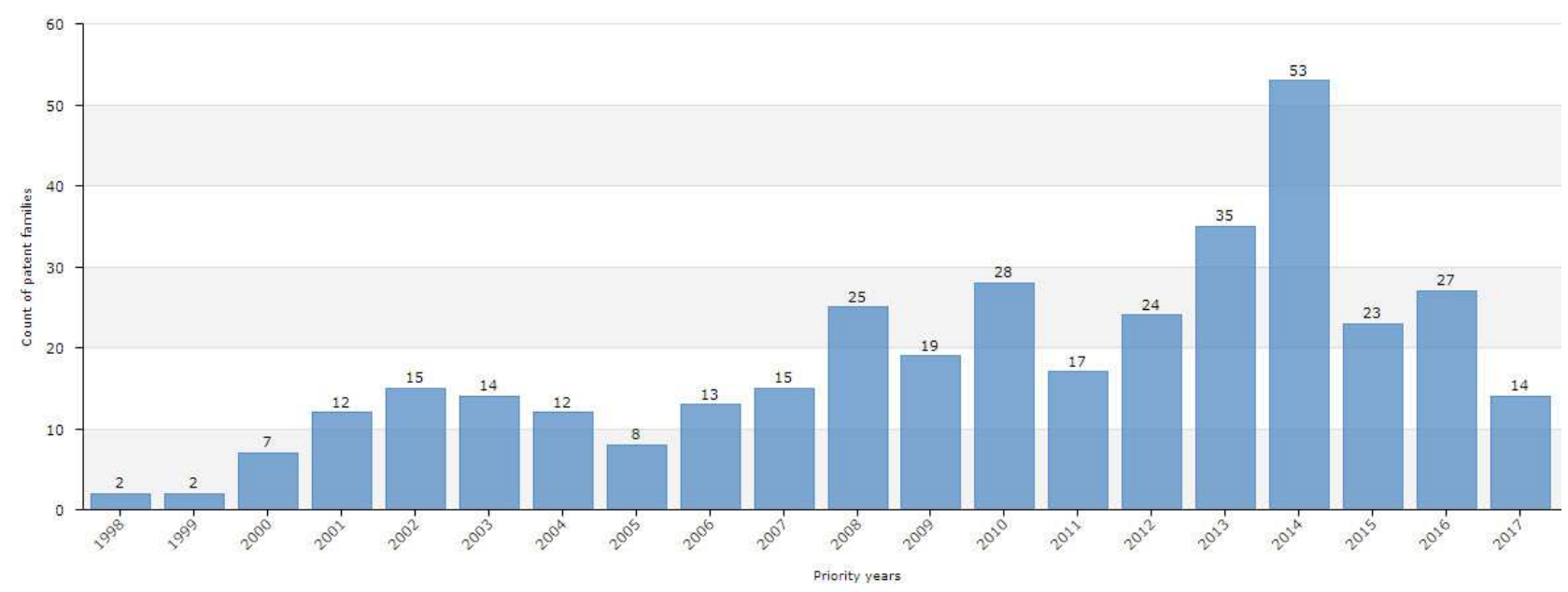

Fonte: Elaborada pelas autoras deste artigo, gerada no software Questel Orbit@ (ORBIT INTELLIGENCE, 2018b)

A Figura 9 mostra que os principais centros de pesquisa na área estão concentrados nos Estados Unidos e na China, seguindo a tendência dos resultados das buscas realizadas anteriormente dentro do escopo deste trabalho. Todavia, a concentração maior está na empresa China Sinopec, que possui 64 patentes depositadas, e de outras que aparecem com número de depósitos menores. 
Figura 9 - Concentração das atividades de P\&D na área de syngas e catálise
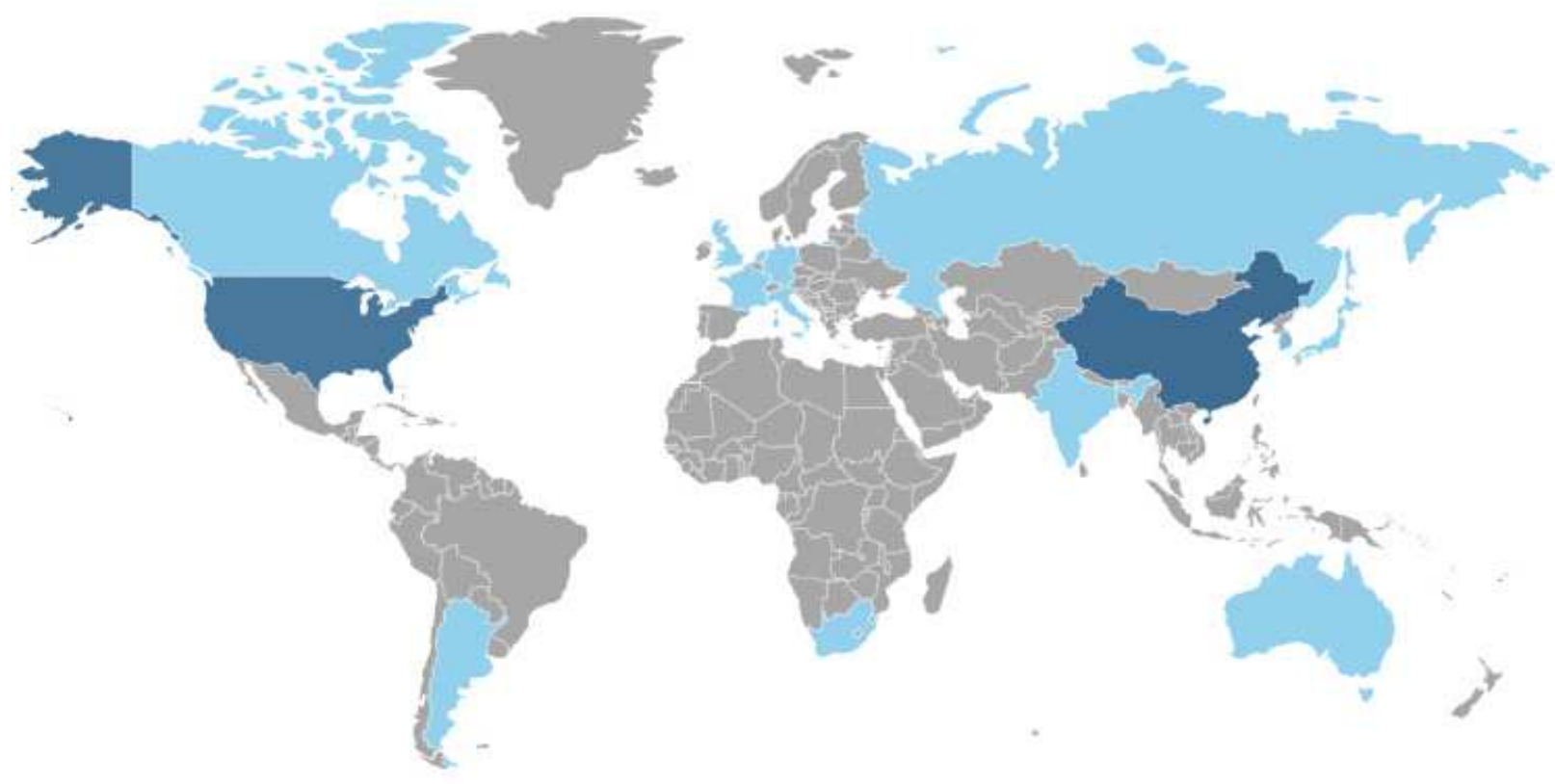

1

122

Fonte: Elaborada pelas autoras deste artigo, gerada no software Questel Orbit ${ }^{\circledR}$ (ORBIT INTELLIGENCE, 2018b)

A incipiência das pesquisas na área de syngas e catálise é evidenciada pela relação entre o número de patentes depositadas e o número de artigos publicados. A Figura 10 mostra quanto a maturidade das pesquisas nessa área é alta, ao passo que a maturidade tecnológica ainda é bem inferior. Tal informação indica que as pesquisas estão avançadas, mas o desenvolvimento tecnológico visando à aplicação industrial ainda carece de mais investimentos.

Figura 10 - Quantidade de depósito de patentes e de artigos nos últimos 20 anos

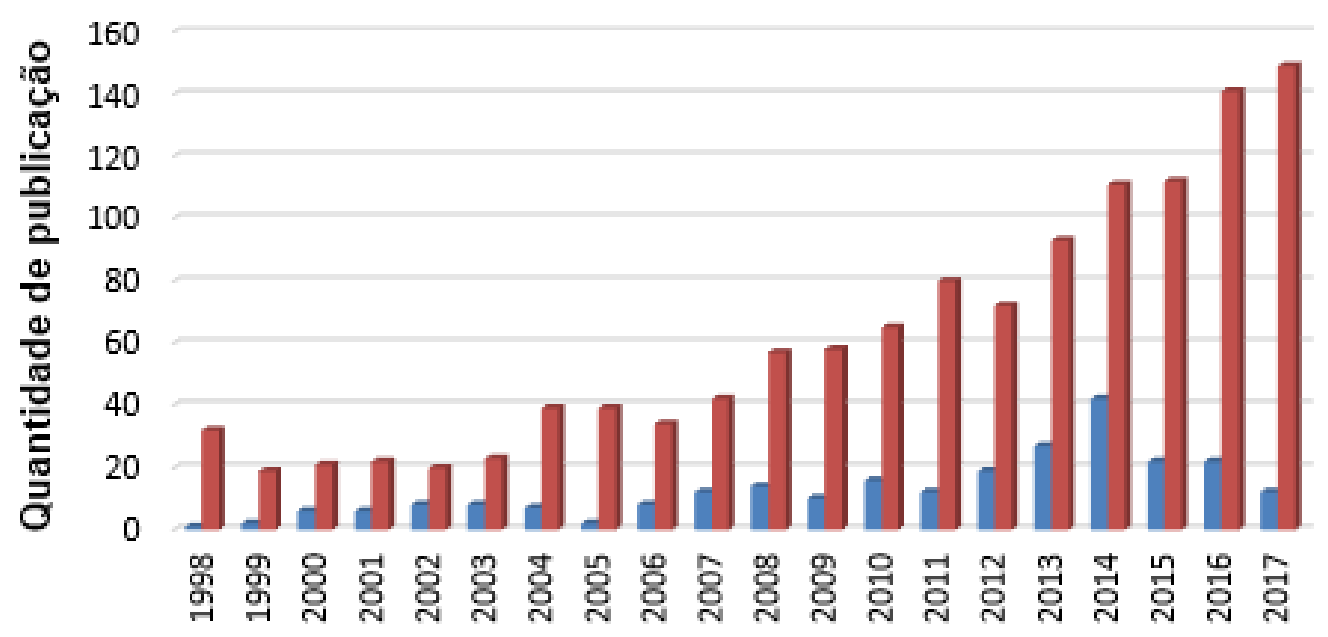

\section{Anos de publicação}

- PATENTES ARTIGOS

Fonte: Elaborada pelas autoras deste artigo, gerada no software Questel Orbit ${ }^{\circledR}$ (ORBIT INTELLIGENCE, 2018b) 
Destaca-se que o fato de a China obter resultados bastante expressivos em todas as buscas realizadas deve-se em grande medida as suas políticas internas de incentivo ao desenvolvimento de tecnologias verdes. Tais incentivos concentram-se em praticamente todas as áreas passíveis de geração de energia, entre elas as renováveis. Em 2005 a China aprovou sua Renewable Energy Law, que tem o objetivo de "[...] promover o desenvolvimento e a utilização de energia renovável, aumentando o fornecimento, melhorando a estrutura, salvaguardando sua segurança, protegendo o meio ambiente e realizando um desenvolvimento econômico e social sustentável" . Por "energia renovável" entende-se as não fósseis como eólica, solar, hidroenergia, bioenergia, geotérmica e oceânica, entre outras. Além disso, é uma área que merece atenção dos pesquisadores brasileiros, visto o grande potencial nacional no setor, não só em termos de resíduos a serem utilizados como também instituições capacitadas e consolidadas para atuar na área, como a Embrapa Agroenergia.

\section{Considerações Finais}

Atividades de P\&D que gerem propriedade intelectual mostram-se mais consolidadas para a área de biogás, apesar de haver uma mudança de tendência a partir de 2013, indicando boas perspectivas nas áreas de pesquisa sobre syngas e catálise. Para estas, entretanto, a maturidade tecnológica mostra-se baixa, mas com tendência de crescimento e consolidação, tendo em vista o aumento dos incentivos para atividades de P\&D.

As pesquisas mostraram que ambas as áreas têm potencial de crescimento e mercado para serem desenvolvidas. No Brasil, os reatores de biogás são amplamente comercializados; porém, sua aplicação para geração de energia com melhor aproveitamento energético e para geração de outros combustíveis ainda necessita de desenvolvimento tecnológico.

No mundo, muitas empresas estão protegendo tecnologias para geração de energia renovável, mostrando que há mercado futuro para comercialização encorajado por políticas públicas de muitos países, entre eles a China, que apresentou resultados crescentes nas buscas por patentes realizadas.

\section{Referências}

ASENCIOS, Y. J. O. Reações de reforma de biogás sobre catalisadores de NiO-MgO-ZrO2 e NiO-Y2O3-ZrO2. 2013. 122 f. Tese (Doutorado em Química) - Instituto de Química de São Carlos, Universidade de São Paulo, São Carlos, 2013.

BRDAR, D. R.; JONES, M. R. GE IGCC Technology and experience with advanced gas turbines. [2018]. Disponível em: <https:/www.ge.com/content/dam/gepower-pgdp/global/en_US/ documents/technical/ger/ger-4207-ge-igcc-technology-experience-advanced-gas-turbines.pdf > . Acesso em: 6 jul. 2018.

CLARIVATE ANALYTICS. Web of Science: base de dados on-line. [2018]. Disponível em: <https:// login.webofknowledge.com/error/Error?PathInfo=\%2F\&Error=IPError $>$. Acesso em: 3 jul. 2018.

DA SILVA, M. I.; DE BORTOLI, A. L. Modelagem e simulação do processo de formação do biogás. Proceeding Series of the Brazilian Society of Computational and Applied Mathematics, São Carlos, v. 6, n. 1, p. 1-7, 2018. 
DOS SANTOS, R. O.; DE SOUSA, S. L.; PRATA, D. M. Simulation and optimization of a methanol synthesis process from different biogas sources. Journal of Cleaner Production, [S.I.], v. 186, p. 821-830, 2018.

DRIF, A. et al. Study of the dry reforming of methane and ethanol using Rh catalysts supported on doped alumina. Applied Catalysis A: General, [S.l.], v. 504, p. 576-584, 2015.

HERMES, N. A. Hidrogênio e nanotubos de carbono por decomposição catalítica do metano: desempenho de catalisadores à base de cobalto e alumínio. 2010. 93 f. Dissertação (Mestrado em Engenharia Química) - Programa de Pós-Graduação em Engenharia Química, Universidade Federal do Rio Grande do Sul, Porto Alegre, 2010.

KADAM, R.; PANWAR, N. L. Recent advancement in biogas enrichment and its applications. Renewable and Sustainable Energy Reviews, [S.1.], v. 73, p. 892-903, 2017.

LI, D. et al. Carbon dioxide reforming of methane over Ru catalysts supported on Mg-Al oxides: A highly dispersed and stable $\mathrm{Ru} / \mathrm{Mg}(\mathrm{Al}) \mathrm{O}$ catalyst. Applied Catalysis B: Environmental, [S.l.], v. 200, p. 566-577, 2017.

MINISTRY OF COMMERCE PEOPLE'S REPUBLIC OF CHINA. Renewable energy law of the people's Republico f China. 2013. Disponivel em: < http://english.mofcom.gov.cn/article/ policyrelease/Businessregulations/201312/20131200432160.shtml>. Acesso em: 6 jul. 2018.

MORAL, A. et al. Syngas production by means of biogas catalytic partial oxidation and dry reforming using Rh-based catalysts. Catalysis Today, [S.I.], v. 299, p. 280-288, 2018.

ORBIT INTELLIGENCE. Base de dados on-line. [2018a]. Disponível em: <ttps://www.orbit. com/>. Acesso em: $17 \mathrm{dez} .2018$.

ORBIT INTELLIGENCE. Questel software. [2018b]. Disponível em: <https://www.questel.com/> . Acesso em: 17 dez. 2018.

PHAN, T. S. et al. Hydroxyapatite supported bimetallic cobalt and nickel catalysts for syngas production from dry reforming of methane. Applied Catalysis B: Environmental, [S.I.], v. 224, p. 310-321, 2018.

SCHULTZ, E.; SOARES, I. Reforma do biogás: revisão. 2014. Brasília, DF: Embrapa Agroenergia, 2014. Circular Técnica, 13.

SERRANO-LOTINA, A. et al. Biogas reforming on La-promoted NiMgAl catalysts derived from hydrotalcite-like precursors. Journal of Power Sources, [S.l.], v. 196, n. 9, p. 4404-4410, 2011.

TEIXEIRA, L. P. Prospecção Tecnológica: importância, métodos e experiências da Embrapa Cerrados. 2013. Disponível em: <https://www.infoteca.cnptia.embrapa.br/bitstream/doc/981247/1/ doc317.pdf>. Acesso em: 6 jul. 2018.

US DEPARTMENT OF ENERGY (NETL). Entrained flow gasifiers. GE Energy (Formerly Chevron Texaco) Gasifier. [2018]. Disponível em: <https:/www.netl.doe.gov/research/coal/energy-systems/ gasification/gasitfipedia/ge >. Acesso em: 6 jul. 2018.

VITA, A. et al. Methanol synthesis from biogas: a thermodynamic analysis. Renewable Energy, [S.l.], v. 118, p. 673-684, 2018. 
YAO, X. et al. Influence of different supports on the physicochemical properties and denitration performance of the supported Mn-based catalysts for NH3-SCR at low temperature. Applied Surface Science, [S.l.], v. 402, p. 208-217, 2017.

ZENG, Y. X. et al. Low temperature reforming of biogas over K-, Mg-and Ce-promoted Ni/Al2O3 catalysts for the production of hydrogen rich syngas: Understanding the plasma-catalytic synergy. Applied Catalysis B: Environmental, [S.I.], v. 224, p. 469-478, 2018.

\section{Sobre as Autoras}

\section{Munique Gonçalves Guimarães}

E-mail: muniquegg@gmail.com

Formação: Doutoranda em Tecnologias Química e Biológica, pela Universidade de Brasília no Laboratório de Bioprocessos Cervejeiros e Catálise Aplicada a Energias Renováveis (LaBCCERva) - IQ/UnB; mestra em Química, pela Universidade de Brasília (UnB); e bacharel em Química Tecnológica, pela UnB.

Endereço profissional: Universidade de Brasília, Instituto de Química, Laboratório B1-75/31. Setor Campus Universitário Darcy Ribeiro, Asa Norte - Brasília, DF. CEP: 70904-970.

\section{Grace Ferreira Ghesti}

E-mail: ghesti.grace@gmail.com

Formação: Doutora em Química, pela Universidade de Brasília (UnB); mestra em Certified Brewmaster Course Versuchs- und Lehranstalt für Brauerei, pela VLB Berlin, Alemanha, e em Química, pela UnB; e bacharela em Química, pela UnB.

Endereço profissional: Universidade de Brasília, Instituto de Química (IQ). Setor Campus Universitário Darcy Ribeiro, Asa Norte - Brasília, DF. CEP: 70297-400.

\section{Camila Lisdalia Dantas Ferreira}

E-mail: camilaferreira.ri@gmail.com

Formação: Mestra em Propriedade Intelectual e Transferência de Tecnologia para Inovação, pela Universidade de Brasília (UnB); e bacharela em Relações Internacionais, pelo Centro Universitário de Brasília (UniCeub).

Endereço profissional: SHIS Quadra 6, Bloco A, sala 805, Complexo Brasil 21, Asa Sul - Brasília, DF. CEP: 70297-400. 